\title{
INFLUÊNCIA DO FENÔMENO ENOS NAS ONDAS DE FRIO E EPISÓDIOS DE GEADAS EM SANTA CATARINA
}

\author{
Maikon Passos Amilton Alves ${ }^{(a)}$, Rosandro Boligon Minuzzi ${ }^{(b)}$ Rafael Brito Silveira ${ }^{(c)}$ \\ (a) Doutorando do Progama de Pós-Graduação em Geografia da Universiade Federal de Santa Catarina (UFSC), \\ maiconpassos@gmail.com \\ (b) Professor Doutor da Universidade Federal de Santa Catarina (UFSC), Centro de Ciências Agrárias, \\ rbminuzzi@hotmail.com \\ (c) Doutorando do Progama de Pós-Graduação em Geografia da Universiade Federal de Santa Catarina (UFSC), \\ rafael.brito@posgrad.ufsc.br
}

\section{Eixo: Climatologia em Diferentes Níveis Escalares: Mudanças e Variabilidades}

\begin{abstract}
Resumo/
Este trabalho avaliou a relação entre o ENOS, às ondas de frio e as ocorrências de geadas em Santa Catarina durante o trimestre invernal. Considerou-se onda de frio o evento em que a temperatura média diária do ar na superfície estava pelo menos duas vezes o desvio padrão abaixo do valor médio da série, naquele dia, durante dois dias consecutivos ou mais. O ENOS foi submetido à análise estatística através dos testes de significância para as médias (test t Student) e variâncias (teste F), com um nível de significância de $\alpha=5 \%$. Constatou-se que o ENOS apresenta relação nas ocorrências de ondas de frio (Itapiranga e Lages) e com os episódios de geada (Itajaí, Chapecó e Ituporanga) em Santa Catarina.
\end{abstract}

Palavras chave: Ondas de frio; Geada; ENOS; Santa Catarina.

\section{Introdução}

Em Santa Catarina (figura 1), é comum no início do outono ocorrer às primeiras incursões de massas de ar polares, as quais provocam o declínio das temperaturas, além de favorecer a formação de geadas, principalmente nas regiões do Planalto e Oeste do estado. Mas, é no período invernal que o anticiclone polar se desloca mais sobre a Argentina em direção ao sul do Brasil, invadindo o território catarinense e provocando acentuada queda de temperatura e ventos fortes do quadrante sul (MONTEIRO, 2001). 


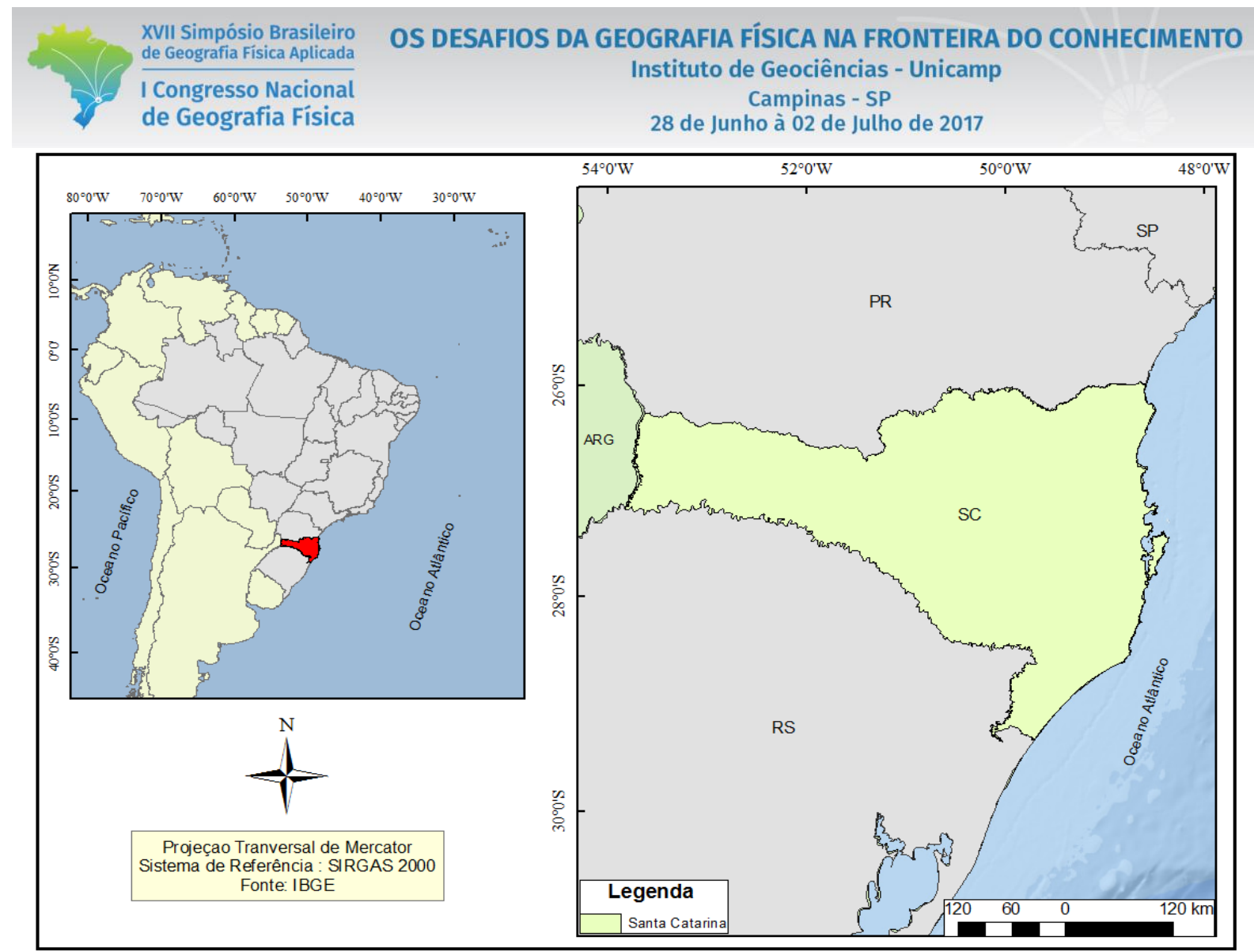

Figura 1 - Mapa de localização do estado de Santa Catarina - Brasil. Elaborado por Maikon P. A. Alves.

Não há consenso na definição de onda de frio. Encontram-se várias formas de defini-la como nos trabalhos de Boyle, (1986); Konrad, (1996); Walsh et al., (2001); Escobar, (2007); Vavrus et. al., (2006); Firpo, (2008). No Manual de Desastres Naturais Castro et al. (1995, p.21) caracterizam uma onda de frio por um evento de rápida e grande queda na temperatura, sobre uma extensa área, permanecendo por varias horas, dias e por vezes uma semana ou mais. Segundo este trabalho, na América do Sul as ondas de frio são mais recorrentes entre os meses de maio e setembro, com predominância nos meses invernais de julho e agosto; normalmente os episódios duram de quatro a cinco dias.

Para Vavrus et al. (2006) um extremo de frio é definido como uma ocorrência de dois dias ou mais consecutivos, durante o qual a temperatura média diária do ar na superfície, é pelo menos duas vezes o desvio padrão abaixo da temperatura média do local de inverno.

As ondas de frios diferem das geadas ou esfriamentos locais, pois abrangem grandes porções da atmosfera. A atmosfera durante as ondas de frio caracteriza-se por pressões atmosféricas anormalmente altas, céu claro, redução ou substituição da precipitação líquida e período de geadas e temperaturas anormalmente baixas (MENDONÇA; ROMERO, 2012).

A geada é um fenômeno meteorológico registrado visualmente pelos observadores, geralmente nas estações convencionais, ou pode ser estimada a sua ocorrência mediante análise de outras variáveis 
meteorológicas registradas como a temperatura em relva (ou do solo), temperatura do ar horária e, ou, temperatura mínima diária, umidade relativa do ar, molhamento foliar, etc (MINUZZI, 2010).

Além dos fatores geográficos como latitude, relevo, altitude, continentalidade e maritimidade, que acabam influenciando as temperaturas no estado (AGUIAR; MENDONÇA, 2004) há também que ser considerado o impacto das variações nos padrões atmosféricos, como as variabilidades climáticas de baixa frequência. O El Niño-Oscilação Sul (ENOS) é um dos fenômenos mais estudados, por produzirem mudanças na circulação atmosférica que afetam o tempo e o clima a nível global.

O ENOS é caracterizado por alterações ou anomalias da temperatura de superfície do mar (TSM) na região do Pacífico Equatorial, nas proximidades da Costa Oeste da América do Sul e que afeta o tempo e o clima em diversos locais do mundo (GRIMM et al., 1998). Apresenta duas fases, a fase quente ou positiva chamada de El Niño e a fase fria ou negativa chamada de La Niña (GRIMM et al., 1998, MOLION, 2007). Segundo Grimm et al.(1998) as fases extremas (El Niño e La Niña) do ENOS provocam significativas alterações nos totais de precipitação mensais e sazonais no Sul do Brasil. Além de produzirem alterações na precipitação, o ENOS, produz perturbações também no campo de temperatura na superfície (GRIMM, TOGATLIAN, 2002; CPTEC/INPE, 2014). As maiores e mais consistentes perturbações sazonais na temperatura na superfície durante a atuação de eventos de EN e LN, ocorrem principalmente durante o inverno (junho, julho e agosto), com temperaturas acima do normal em quase todo o Cone Sul durante eventos de EN e abaixo do normal durante a ocorrência da LN (GRIMM, TOGATLIAN, 2002).

Assim, este trabalho tem como objetivo principal, analisar a possível influência do ENOS em suas diferentes fases nas ocorrências de ondas de frio e nos episódios de geadas no estado catarinense, durante o trimestre invernal.

\section{Material e Métodos}

Os dados e as análises estatisticas demonstrados no decorrer deste artigo, tem como referência o trabalho de Alves (2016), cujo desenvolveu um estudo climatológico sobre as ondas de frio invernais em Santa Catarina, relacionando-as com as variabilidades climáticas de baixa frequência, dentre as quais o El Niño Oscilação Sul (ENOS).

\subsection{Contabilização das ondas de frio e ocorrência de geada}

Foram utilizados dados de temperatura média do ar diária, medida em graus Celsius $\left(\mathrm{C}^{\circ}\right)$, de 11 estações meteorológicas convencionais (figura 2) pertencentes à Empresa de Pesquisa Agropecuária e Extensão Rural de Santa Catarina/Centro de Informações Ambientais e de Hidrometeorologia (Epagri/Ciram) e ao 


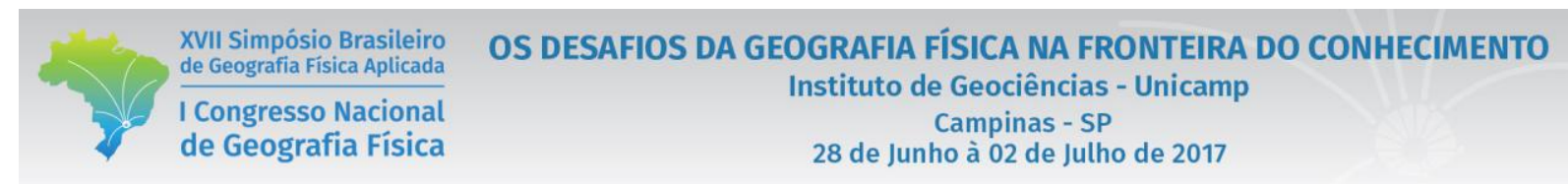

Instituto Nacional de Meteorologia (INMET). A série temporal compreende os anos de 1983 a 2013, no período invernal, totalizando 31 anos de dados. Os registros de geada também foram cedidos pela Epagri/Ciram. Para efeito de regionalização utilizou-se a divisão delimitada pelas Zonas Agroecológicas de Santa Catarina (THOMÉ et al.,1999).

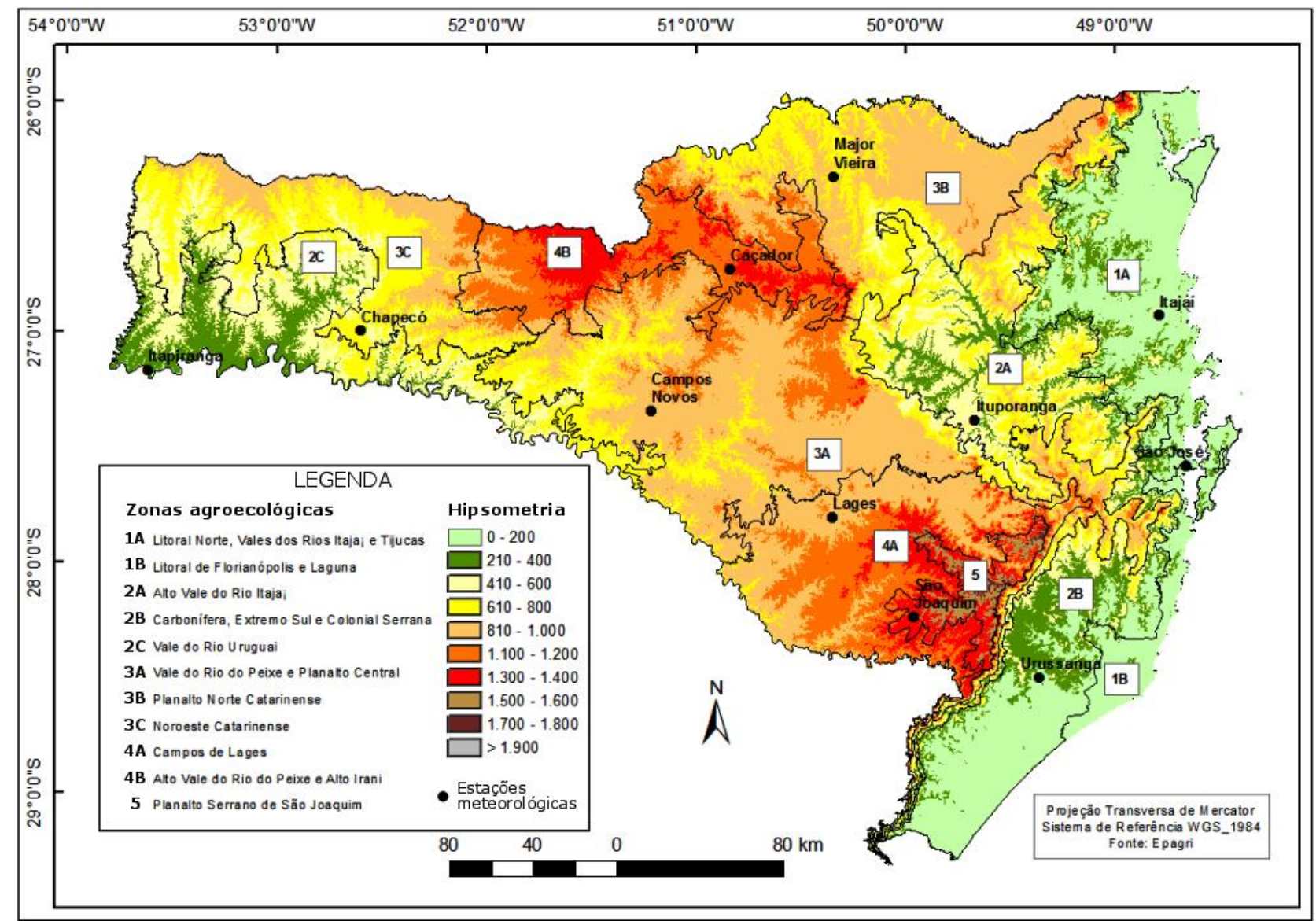

Figura 2 - Mapa hipsométrico do estado de Santa Catarina com a localização das estações meteorológicas e suas respectivas zonas agroecológicas. Elaborado por Maikon P. A. Alves e Rafael Brito Silveira.

Os dados de temperatura média do ar compensada foram tabulados via programa Excel 2007 e então o método para classificação das ondas de frio de Vavrus et al. (2006) foi aplicado. Este método considera como onda de frio o evento no qual a temperatura média diária do ar na superfície esta pelo menos duas vezes o desvio padrão abaixo do valor da média durante dois dias consecutivos ou mais, no período invernal. O desvio padrão (S) é o valor médio dos 365 desvios padrões diário de temperatura do ar. Todavia, este trabalho trata apenas dos 92 dias do período de inverno ( $1^{\circ}$ de junho a 31 de agosto). 


\section{2 - Análises estatísticas relacionadas ao ENOS}

As análises relacionadas aos eventos de ENOS foram efetuadas com base nos dados do Índice Oceanic Niño (ONI) obtidos do Serviço Climatológico Nacional dos Estados Unidos (NOAA- National Weather Service) de acordo com a tabela 1 , que considera como um evento ENOS, o limite de $+/-0,5^{\circ} \mathrm{C}$ para a anomalia da TSM da região Niño 3.4 observadas em pelo menos cinco trimestres.

A tabela 1 apresenta-se dividida em trimestres e os valores médios do ONI, que caracterizam eventos de El Niño (fonte na cor vermelha), La Niña (fonte na cor azul) e período de neutralidade (fonte na cor preta).

Os episódios de EN e LN iniciam no outono ou no inverno austral em um ano (0) e terminam sempre no ano seguinte (+1), com um pico nas anomalias de TSM de novembro a janeiro (TRENBERTH, 1997).

Este trabalho considerou os trimestres de abril/maio/junho até agosto/setembro/outubro por abrangerem pelo menos um dos meses de inverno. Verificou-se a influência de uma das fases do ENOS para cada ano da série de dados (período invernal), de acordo com o ciclo médio EN/LN, sendo o inverno/ano de inicio do evento denominado de ano (0) e o inverno/ano seguinte de ano (+1). Os trimestres (junho/julho/agosto; julho/agosto/setembro e agosto/setembro/outubro) foram considerados no ano (0) e são ponderados de ano (+1) os trimestres (abril/maio/junho e maio/junho/julho).

\begin{tabular}{|c|c|c|c|c|c|c|c|c|c|c|c|c|c|c|}
\hline \multicolumn{3}{|c|}{ Sesson } & IIA & JAS & ASO & $50 N$ & OND & NDJ & DAF & JAM & FMA & MAM & AMJ & MJJ \\
\hline 1982 & - & 1983 & 0.8 & 1 & 1.5 & 19 & 2.1 & 21 & 2.1 & 18 & 15 & 1.2 & 1 & 0.7 \\
\hline 1983 & - & 1984 & 0.3 & 0 & -0.3 & -0.6 & -0.8 & -0.8 & -0.5 & -0.3 & -0.3 & -0.4 & -0.4 & -0.4 \\
\hline 1984 & - & 1985 & -0.3 & -0.2 & -0.3 & -0.6 & -0.9 & -1.1 & -0.9 & -0.7 & -0.7 & -0.7 & -0.7 & -0.6 \\
\hline 1985 & - & 1986 & -0.4 & -0.4 & -0.4 & -0.3 & -0.2 & -0.3 & -0.4 & -0.4 & -0.3 & -0.2 & -0.1 & 0 \\
\hline 1986 & - & 1987 & 0.2 & 0.4 & 0.7 & 0.9 & 1 & 11 & 1.1 & 12 & 11 & 1 & 0.9 & 1.1 \\
\hline 1987 & - & 1988 & 14 & 16 & 1.6 & 14 & 1.2 & 11 & 0.8 & 0.5 & 0.1 & -0.3 & -0.8 & -12 \\
\hline 1988 & - & 1989 & -1.2 & -11 & -12 & -1.4 & -17 & -1.8 & -16 & -1.4 & -11 & -0.9 & -0.6 & -0.4 \\
\hline 1989 & - & 1990 & -0.3 & -0.3 & -0.3 & -0.3 & -0.2 & -0.1 & 0.1 & 0.2 & 0.2 & 0.2 & 0.2 & 0.3 \\
\hline 1990 & - & 1991 & 0.3 & 0.3 & 0.4 & 0.3 & 0.4 & 0.4 & 0.4 & 0.3 & 0.2 & 0.2 & 0.4 & 0.6 \\
\hline 1991 & - & 1992 & 0.7 & 0.7 & 0.7 & 0.8 & 1.2 & 14 & 1.6 & 15 & 14 & 1.2 & 1 & 0.8 \\
\hline 1992 & - & 1993 & 0.5 & 0.2 & 0 & -0.1 & -0.1 & 0 & 0.2 & 0.3 & 0.5 & 0.7 & 0.8 & 0.6 \\
\hline 1993 & - & 1994 & 0.3 & 0.2 & 0.2 & 0.2 & 0.1 & 0.1 & 0.1 & 0.1 & 0.2 & 0.3 & 0.4 & 0.4 \\
\hline 1994 & - & 1995 & 0.4 & 0.4 & 0.4 & 0.6 & 0.9 & 1 & 0.9 & 0.7 & 0.5 & 0.3 & 0.2 & 0 \\
\hline 1995 & - & 1996 & -0.2 & -0.5 & -0.7 & -0.9 & -1 & -0.9 & -0.9 & -0.7 & -0.6 & -0.4 & -0.2 & -0.2 \\
\hline 1996 & - & 1997 & -0.2 & -0.3 & -0.3 & -0.4 & -0.4 & -0.5 & -0.5 & -0.4 & -0.2 & 0.1 & 0.6 & 1 \\
\hline 1997 & - & 1998 & 14 & 17 & 2 & 22 & 2.3 & 23 & 2.1 & 18 & 14 & 1 & 0.5 & -0.1 \\
\hline 1998 & - & 1999 & -0.7 & -1 & -12 & -1.2 & -13 & -1.4 & -14 & -1.2 & -1 & -0.9 & -0.9 & -1 \\
\hline 1999 & - & 2000 & -1 & -1 & -11 & -1.2 & -14 & -1.6 & -16 & -1.4 & -11 & -0.9 & -0.7 & -0.7 \\
\hline 2000 & - & 2001 & -0.6 & -0.5 & -0.6 & -0.7 & -0.8 & -0.8 & -0.7 & -0.6 & -0.5 & -0.3 & -0.2 & -0.1 \\
\hline 2001 & - & 2002 & 0 & -0.1 & -0.1 & -0.2 & -0.3 & -0.3 & -0.2 & -0.1 & 0.1 & 0.2 & 0.4 & 0.7 \\
\hline 2002 & - & 2003 & 0.8 & 0.9 & 1 & 12 & 1.3 & 11 & 0.9 & 0.6 & 0.4 & 0 & -0.2 & -0.1 \\
\hline 2003 & - & 2004 & 0.1 & 0.2 & 0.3 & 0.4 & 0.4 & 0.4 & 0.3 & 0.2 & 0.1 & 0.1 & 0.2 & 0.3 \\
\hline 2004 & - & 2005 & 0.5 & 0.7 & 0.7 & 0.7 & 0.7 & 0.7 & 0.6 & 0.6 & 0.5 & 0.5 & 0.4 & 0.2 \\
\hline 2005 & - & 2006 & 0.1 & 0 & 0 & -0.1 & -0.4 & -0.7 & -0.7 & -0.6 & -0.4 & -0.2 & 0 & 0.1 \\
\hline 2006 & - & 2007 & 0.2 & 0.3 & 0.5 & 0.8 & 0.9 & 1 & 0.7 & 0.3 & 0 & -0.1 & -0.2 & -0.2 \\
\hline 2007 & - & 2008 & -0.3 & -0.6 & -0.8 & -1.1 & -12 & -1.3 & -14 & -1.3 & -11 & -0.9 & -0.7 & -0.5 \\
\hline 2008 & - & 2009 & -0.3 & -0.2 & -0.2 & -0.3 & -0.5 & -0.7 & -0.8 & -0.7 & -0.4 & -0.1 & 0.2 & 0.4 \\
\hline 2009 & - & 2010 & 0.5 & 0.6 & 0.7 & 1 & 1.2 & 13 & 1.3 & 11 & 0.8 & 0.5 & 0 & -0.4 \\
\hline 2010 & - & 2011 & -0.8 & -11 & -13 & -1.4 & -13 & -1.4 & -13 & -1.1 & -0.8 & -0.6 & -0.3 & -0.2 \\
\hline 2011 & - & 2012 & -0.3 & -0.5 & -0.7 & -0.9 & -0.9 & -0.8 & -0.7 & -0.6 & -0.5 & -0.4 & -0.3 & -0.1 \\
\hline 2012 & - & 2013 & 0.1 & 0.3 & 0.4 & 0.4 & 0.2 & -0.2 & -0.4 & -0.5 & -0.3 & -0.2 & -0.2 & -0.2 \\
\hline 2013 & - & 2014 & -0.2 & -0.2 & -0.2 & -0.2 & -0.2 & -0.3 & -0.5 & -0.6 & -0.4 & -0.2 & 0 & 0 \\
\hline 2014 & - & 2015 & 0 & 0 & 0.2 & 0.4 & 0.6 & 0.6 & 0.5 & 0.4 & 0.5 & 0.7 & 0.9 & 1.0 \\
\hline
\end{tabular}

Tabela 1 - Classificação do Índice Oceanic Niño (ONI) para região Niño 3.4 de 1983 e 2013. Fonte: Golden Gate Weather Services (2015). 
Os registros do ENOS foram submetidos à análise estatística através dos testes de significância para as médias e variâncias, com nível de $\alpha=0,05 \%$ de probabilidade de erro. Para comparar as diferenças entre as médias utilizou-se o teste $\mathrm{t}$ de Student, e para a variância foi utilizado o teste $\mathrm{F}$. As análises foram realizadas utilizando o programa estatístico PAST (HAMMER; HARPER; RYAN, 2008).

\section{Resultados e Discussão}

\subsection{Relação das ondas de frio com o ENOS}

Verificou-se a influência do ENOS para cada ano da série de dados (período invernal), de acordo com o ciclo médio El Niño (EN) /La Niña (LN), sendo o inverno/ano de inicio do evento (ano 0) e o inverno/ano seguinte $($ ano +1$)$.

Para Grimm (1998) o efeito de ENOS sobre a temperatura não é tão forte quanto sobre a precipitação. A respeito da implicação do ENOS sobre a temperatura, Barros et al. (2002) detalharam os efeitos sobre o sudeste da América do Sul, incluindo o Sul do Brasil. Referente ao inverno (JJA) durante episódios EN (LN), o efeito mais forte foi constatado no ano (0), com anomalias positivas (negativas) de temperatura nos subtrópicos da América do Sul, com centro no norte da Argentina, estendendo-se sobre a região Sul do Brasil.

\subsubsection{Ondas de frio por Inverno Ano (0)}

Quando aplicado o teste estatístico de significância para as médias (teste $\mathrm{t}$ de Student), com nível de significância de 5\% (tabela 2) percebe-se que o número médio de ondas de frio em anos neutros é superior em grande parte das estações analisadas, exceção Itajaí, Campos Novos, Lages e São Joaquim onde as maiores ocorrências foram em anos de La Niña. Nenhuma estação apresentou um número médio de ondas de frio maior durante El Niño. No entanto essas médias não diferem significativamente, ou seja, para este estudo não se pode afirmar que há relação entre o número médio de ondas de frio e ENOS para o inverno de início (ano 0).

Tabela 2 - Valores médios do número de ondas de frio nas fases do ENOS (Ano 0) para Santa Catarina no período invernal de 1983 - 2013.

\begin{tabular}{|c|c|c|c|c|c|c|c|c|c|c|c|}
\hline \multirow{2}{*}{ Fase do ENOS- } & \multicolumn{11}{|c|}{ Estaçōes Meteorológ̨icas } \\
\hline & Itajai & São José & Ituporanga & Urussanga & Itapiranga & Campos Nowos & MajorVieira & Chapecó & Lages & Caçador & Sãoloaquim \\
\hline EN & $1,12 \mathrm{a}$ & $0,78 \mathrm{a}$ & $2,21 \mathrm{a}$ & $1,14 a$ & $0,61 a$ & $1,41 \mathrm{a}$ & $2,26 \mathrm{a}$ & $1,41 \mathrm{a}$ & $2,00 \mathrm{a}$ & $1,92 \mathrm{a}$ & $1,69 a$ \\
\hline LN & $3,26 \mathrm{a}$ & $2,50 \mathrm{a}$ & $1,92 \mathrm{a}$ & $0,83 \mathrm{a}$ & 1,69 a & $2,55 \mathrm{a}$ & $2,69 \mathrm{a}$ & $3,36 \mathrm{a}$ & $2,78 b$ & $3,14 \mathrm{a}$ & $2,83 \mathrm{a}$ \\
\hline Neutro & $2,00 \mathrm{a}$ & $2,55 \mathrm{a}$ & $1,80 \mathrm{a}$ & $2,35 a$ & $1,65 a$ & $2,25 \mathrm{a}$ & $3,11 \mathrm{a}$ & $1,63 a$ & $0,71 \mathrm{a}$ & $1,69 \mathrm{a}$ & $1,80 \mathrm{a}$ \\
\hline
\end{tabular}

Nota: Médias seguidas por letras iguais nas colunas não diferem entre si pelo teste t (Student) ao nível de 5\% de significância. Fonte: Elaborado pelos autores. 
OS DESAFIOS DA GEOGRAFIA FÍSICA NA FRONTEIRA DO CONHECIMENTO

Instituto de Geociências - Unicamp

Campinas - SP

28 de Junho à 02 de Julho de 2017

Na tabela 3, apresentam-se os resultados do teste de significância para as variâncias, através do teste F, com nível de significância de 5\%. A variação das ocorrências de ondas de frio é maior na fase negativa do ENOS (La Niña) em oito das onze estações. Atente-se para a estação de Lages, a qual apresenta diferenças estatísticas entre os valores médios das variâncias, o que sugere que em anos de La Niña $(2,78)$ tem-se uma maior variação no número de ondas de frio em relação aos anos considerados neutros $(0,71)$ ou sobre influencia do E1 Niño. O que vem ao encontro das considerações realizadas por Marengo e Oliveira (1998) que ao estudarem eventos de La Niña ocorridos no passado, observaram uma maior variabilidade das condições de tempo e clima em relação aos eventos de El Niño.

Tabela 3 - Resultados das variâncias para os números de ondas de frio nas fases do ENOS (Ano 0) para Santa Catarina no período invernal de 1983 - 2013.

\begin{tabular}{|c|c|c|c|c|c|c|c|c|c|c|c|}
\hline \multirow{2}{*}{ Fase do ENOS } & \multicolumn{11}{|c|}{ Estaçōes Meteoroloǵg̨icas } \\
\hline & Itajai & São José & |tuporanga ! & Urussanga & Itapiranga & Campos Nowos & MajorVieira & Chapecó & Lages & Cacador. & São Joaquim \\
\hline EN & $1,12 \mathrm{a}$ & $0,78 \mathrm{a}$ & $2,21 \mathrm{a}$ & $1,14 \mathrm{a}$ & $0,61 \mathrm{a}$ & 1,41a & $2,26 \mathrm{a}$ & $1,41 \mathrm{a}$ & $2,00 \mathrm{a}$ & $1,92 \mathrm{a}$ & 1,69 a \\
\hline IN & $3,26 \mathrm{a}$ & $2,50 \mathrm{a}$ & 1,92 a & $0,83 \mathrm{a}$ & 1,69 a & $2,55 a$ & $2,69 \mathrm{a}$ & $3,36 \mathrm{a}$ & $2,78 \mathrm{~b}$ & 3,14a & $2,83 \mathrm{a}$ \\
\hline Neutro & $2,00 \mathrm{a}$ & 2,55 a & $1,80 \mathrm{a}$ & $2,35 \mathrm{a}$ & $1,65 \mathrm{a}$ & $2,25 \mathrm{a}$ & 3,11 a & $1,63 \mathrm{a}$ & $0,71 \mathrm{a}$ & 1,69a & 1,80 \\
\hline
\end{tabular}

Nota: Variâncias seguidas por letras iguais nas colunas não diferem entre si pelo teste $\mathrm{F}$ ao nível de 5\% de significância. Fonte: Elaborado pelos autores.

\subsubsection{Ondas de frio por Inverno Ano (+1)}

Ao contrario do ano inicial do ENOS, onde o número médio de ondas de frio em anos neutros é superior em grande parte das estações analisadas, no ano final predominaram os maiores valores durante a La Niña (tabela 4). Resultado semelhante ao encontrado por Firpo et al.(2012) que concluíram pela influência defasada dos eventos de La Niña sobre temperaturas abaixo da média no Estado do Rio Grande do Sul. Os valores médios da estação de Itapiranga foram os únicos a diferirem estatisticamente entre si, pressupondo que em anos de El Niño tenhamos menores registros de ondas de frio se comparados às condições de normalidade.

Tabela 4 - Valores médios do número de ondas de frio nas fases do ENOS $($ Ano +1) para Santa Catarina no período invernal de 1983 - 2013.

\begin{tabular}{|c|c|c|c|c|c|c|c|c|c|c|c|}
\hline \multirow[t]{2}{*}{ Fase do ENOS } & \multicolumn{11}{|c|}{ Estạ̣es Meteoroloǵgicas } \\
\hline & Itajaj & São José & Ituporanga & Urussanga I & Itapiranga C & Campos Novos & Major Vieira & Ghapecó & lages & Cacador & Săo Joaquin \\
\hline EN & $1,77 \mathrm{a}$ & $1,11 \mathrm{a}$ & $2,12 \mathrm{a}$ & 1,33a & $1,00 \mathrm{~b}$ & $2,62 \mathrm{a}$ & $1,28 \mathrm{a}$ & $3,22 \mathrm{a}$ & $2,00 \mathrm{a}$ & $2,22 \mathrm{a}$ & \\
\hline LN & $2,57 \mathrm{a}$ & $2,00 \mathrm{a}$ & $2,00 \mathrm{a}$ & 1,57 a & $2,00 \mathrm{a}$ & $2,28 \mathrm{a}$ & 2,50 a & $3,28 \mathrm{a}$ & $2,42 a$ & $3,00 \mathrm{a}$ & 3,00 \\
\hline Neutro & $1,60 \mathrm{a}$ & $1,86 \mathrm{a}$ & $1,78 \mathrm{a}$ & $2,06 \mathrm{a}$ & 2,15 a & $2,42 \mathrm{a}$ & $2,00 \mathrm{a}$ & 2,73a & 1,93a & $2,33 \mathrm{a}$ & 2,2 \\
\hline
\end{tabular}

Nota: Médias seguidas por letras iguais nas colunas não diferem entre si pelo teste t (Student) ao nível de 5\% de significância. Fonte: Elaborado pelos autores. 
OS DESAFIOS DA GEOGRAFIA FÍSICA NA FRONTEIRA DO CONHECIMENTO

Instituto de Geociências - Unicamp

Campinas - SP

28 de Junho à 02 de Julho de 2017

Os resultados do teste F para as variâncias na fase final do ENOS no período invernal (ano +1), também demonstram que na fase negativa (La Niña) ocorre uma maior variação nas ocorrências das ondas de frio, em grande parte das estações (tabela 5). Lages foi à estação que apresentou significância estatística de 5\% em relação a suas variâncias. Assim como tinha ocorrido durante os testes para o ano inicial, em anos de La Niña espera-se maior dispersão no número médio de ondas de frio para a região de Lages.

Tabela 5 - Resultados das variâncias para os números de ondas de frio nas fases do ENOS (Ano+1) para Santa Catarina no período invernal de 1983 - 2013.

\begin{tabular}{|c|c|c|c|c|c|c|c|c|c|c|c|}
\hline \multirow{2}{*}{ Fase do ENOS } & \multicolumn{11}{|c|}{ Estap̧es Meteorol ógicas } \\
\hline & Itajai & Sãolosé & |tuporanga & Urussanga & Itapiranga C & ampos Novos 1 & Major Vieira & Chapecó & Lages & Caçador & Sãoloaquim \\
\hline EN & $1,44 a$ & $0,86 a$ & $2,12 a$ & $1,25 a$ & $1,14 a$ & $2,26 a$ & $2,57 a$ & $2,94 a$ & $2,00 a$ & $2,19 \mathrm{a}$ & $1,83 a$ \\
\hline LN & $2,28 \mathrm{a}$ & $2,00 \mathrm{a}$ & $1,66 a$ & 0,61 a & $1,60 a$ & $3,23 a$ & $1,90 a$ & $2,23 \mathrm{a}$ & $2,16 b$ & $2,66 \mathrm{a}$ & $2,00 a$ \\
\hline Neutro & $2,25 \mathrm{a}$ & $2,69 \mathrm{a}$ & $2,02 a$ & $2,20 \mathrm{a}$ & $1,30 \mathrm{a}$ & $1,49 a$ & $3,16 \mathrm{a}$ & $1,35 \mathrm{a}$ & $0,78 a$ & $1,66 \mathrm{a}$ & $2,17 \mathrm{a}$ \\
\hline
\end{tabular}

Nota: Variâncias seguidas por letras iguais nas colunas não diferem entre si pelo teste $\mathrm{F}$ ao nível de $5 \%$ de significância. Fonte: Elaborado pelos autores.

\subsection{Relação dos episódios de geadas com o ENOS}

$\mathrm{Da}$ mesma forma que nas ocorrências de ondas de frio, para os registros de geadas verificou-se a influência do ENOS para cada ano da série de dados (período invernal), de acordo com o ciclo médio El Niño (EN) /La Niña (LN), sendo o inverno/ano de inicio do evento (ano 0) e o inverno/ano seguinte (ano $+1)$.

\subsubsection{Geadas no Inverno Ano (0)}

Ao testar as diferenças entre os valores médios de ocorrências de geada para fase inicial do ENOS (tabela

6), constatou-se que as médias não diferem entre si em nenhuma das estações, ou em outras palavras, as ocorrências médias de geada em Santa Catarina são iguais entre todas as fases do ENOS em seu período inicial.

Tabela 6- Valores médios das ocorrências de geadas durante as ondas de frio nas fases do ENOS (Ano 0) para Santa Catarina no período invernal de 1983 - 2013.

\begin{tabular}{|c|c|c|c|c|c|c|c|c|c|c|c|}
\hline \multirow{2}{*}{ Fae do ENOS } & \multicolumn{11}{|c|}{ Estap̧es Metearologęcas } \\
\hline & |tajai & São José & tupoanga & Unussangु & Itapiranga & Campos Novos & Najor Vieira & Chapecó & lages & Caçador & São loaquim \\
\hline EN & $0,62 \mathrm{a}$ & Q,50: & $3,00 \mathrm{a}$ & $2,5 a$ & $1,14 a$ & $3,62 a$ & $3,16 a$ & $2,87 \mathrm{a}$ & $3,75 a$ & $4,12 a$ & $8,00 \mathrm{a}$ \\
\hline W & $1,25 a$ & $1,5:$ & $1,87 \mathrm{a}$ & $2,50 \mathrm{a}$ & $1,25 a$ & $4,50 \mathrm{a}$ & $4,25 a$ & 3,25 a & $3,37 \mathrm{a}$ & $4,25 \mathrm{a}$ & $5,00 \mathrm{a}$ \\
\hline Neutro & $0,33 \mathrm{a}$ & 0,6 : & $2,50 \mathrm{a}$ & $2,6 a$ & $1,25 a$ & $2,76 \mathrm{a}$ & 4,41a & $3,06 \mathrm{a}$ & $3,40 \mathrm{a}$ & $3,73 \mathrm{a}$ & $1400 \mathrm{a}$ \\
\hline
\end{tabular}

Nota: Médias seguidas por letras iguais nas colunas não diferem entre si pelo teste $\mathrm{t}$ (Student) ao nível de 5\% de significância. Fonte: Elaborado pelos autores. 
Durante a atuação da La Niña, a dispersão no número médio de geada foi maior em quase todas as estações, exceto Ituporanga em fase de El Niño e São Joaquim em anos neutros (tabela 7). As variâncias das estações de Itajaí, São José e Chapecó, são significativas estatisticamente, possuindo valores elevados sob predomínio da La Niña. Já as estações de Ituporanga e novamente Itajaí, têm suas variâncias significativas a 5\%, ao compararmos os valores entre as condições de El Niño e neutralidade. Isto demonstra que durante o evento E1 Niño é de se esperar uma grande variação do número de ocorrências de geadas para Ituporanga e Itajaí, e durante a La Niña maior dispersão nas estações de São José, Itajaí e Chapecó.

Tabela 7 - Resultados das variâncias para os números das ocorrências de geadas durante as ondas de frio nas fases do ENOS (Ano 0) para Santa Catarina no período invernal de 1983 - 2013.

\begin{tabular}{|c|c|c|c|c|c|c|c|c|c|c|c|}
\hline \multirow{2}{*}{ Fare do ENOS } & \multicolumn{11}{|c|}{ Estap̣ōes Metearolog̨icas } \\
\hline & $\mid t a j a j$ & São lose & ltupoanga & Unussangeg & Itapiranga $C$ & ampos Novos & Najor Vieira & Chapeco & Lages & Cacador & Solo Joaquim \\
\hline EN & 198 & $0,57 \%$ & $10,0 \mathrm{~b}$ & $5,0,0$ & $2,14 a$ & $2,55 a$ & $13,76 \mathrm{a}$ & $3,26 \mathrm{a}$ & $5,64 \mathrm{a}$ & $9,55 \mathrm{a}$ & $8,12 a$ \\
\hline LN & 7,646 & $5,92 b$ & $3,26 \mathrm{a}$ & 6,7 & $2,21 a$ & 15,71a & $18,50 \mathrm{a}$ & $9,07 \mathrm{~b}$ & $9,12 \mathrm{a}$ & $11,92 \mathrm{a}$ & $1428 \mathrm{a}$ \\
\hline Neuto & 0,52 & 0,20 & $1,90 \mathrm{a}$ & 8,8 & 3,11a & $5,35 \mathrm{a}$ & $10,81 \mathrm{a}$ & $2,63 \mathrm{a}$ & 4,11a & $4,35 \mathrm{a}$ & $10,70 \mathrm{a}$ \\
\hline
\end{tabular}

Nota: Variâncias seguidas por letras iguais nas colunas não diferem entre si pelo teste $\mathrm{F}$ ao nível de 5\% de significância. Fonte: Elaborado pelos autores.

\subsubsection{Geadas no Inverno Ano (+1)}

$\mathrm{Na}$ fase final do fenômeno ENOS durante o período invernal, as maiores médias relacionadas às ocorrências de geada encontraram-se durante o predomínio da La Niña, exceção feita a Ituporanga que foi sobre a interferência do El Niño (tabela 8). As médias não diferem entre si em nenhuma das estações no ano final. Em outras palavras, as ocorrências médias de geada em Santa Catarina são iguais entre todas as fases do ENOS em seu período final e também na fase inicial como encontrado anteriormente.

Tabela 8 - Valores médios das ocorrências de geadas durante as ondas de frio nas fases do ENOS (Ano+1) para Santa Catarina no período invernal de 1983 - 2013.

\begin{tabular}{|c|c|c|c|c|c|c|c|c|c|c|c|}
\hline \multirow{2}{*}{ Fase do ENOS } & \multicolumn{11}{|c|}{ Estaçōes Meteorológicas } \\
\hline & Itajaj & São José & Ituporanga U & Jussanga & tapiranga & Campos Nowos & Major Vieira & Chapecó & Lages & Caçador & São Joaquim \\
\hline EN & $0,55 a$ & $0,55 \mathrm{a}$ & $2,75 a$ & $2,22 a$ & $0,87 a$ & $3,12 \mathrm{a}$ & $2,71 a$ & $2,66 a$ & $3,33 \mathrm{a}$ & $3,55 a$ & $3,00 a$ \\
\hline LN & $1,57 \mathrm{a}$ & $1,42 \mathrm{a}$ & $2,42 \mathrm{a}$ & $2,71 \mathrm{a}$ & $2,16 a$ & $4,57 a$ & $5,50 \mathrm{a}$ & $4,14 a$ & $3,57 \mathrm{a}$ & $4,57 \mathrm{a}$ & $5,14 a$ \\
\hline Neutro & $0,26 \mathrm{a}$ & $0,20 \mathrm{a}$ & $2,14 a$ & $2,46 a$ & $1,00 \mathrm{a}$ & $3,14 a$ & $4,15 a$ & $2,80 \mathrm{a}$ & $3,53 \mathrm{a}$ & $3,93 \mathrm{a}$ & $4,26 \mathrm{a}$ \\
\hline
\end{tabular}

Nota: Médias seguidas por letras iguais nas colunas não diferem entre si pelo teste $\mathrm{t}$ (Student) ao nível de 5\% de significância. Fonte: Elaborado pelos autores.

A partir do teste de significância para as variâncias (teste F) no inverno de término do fenômeno ENOS (tabela 9), encontrou-se os mesmos resultados do período de início (ano 0). As variâncias das estações de Itajaí, São José e Chapecó, são significativas estatisticamente, possuindo valores elevados sobre 
predomínio da La Niña. Itajaí também tem suas médias diferindo em condições de El Niño, assim como Ituporanga ao compararmos os valores entre as condições de El Niño $(10,78)$ e neutralidade $(2,28)$.

Tabela 9 - Resultados da variância para os números das ocorrências de geadas durante as ondas de frio nas fases do ENOS (Ano+1) para Santa Catarina no período invernal de 1983 - 2013.

\begin{tabular}{c|ccccccccccc|}
\hline \multirow{2}{*}{ Fase do ENOSOS } & \multicolumn{10}{|c}{ Estaçōes Meteorológicas } \\
\cline { 2 - 11 } & Itajai & São José Ituporanga Urussanga Itapiranga Campos Nowos Major Vieira Chapecó & Lages & Caçador São Joaquim \\
\hline EN & $1,77 \mathrm{C}$ & $0,52 \mathrm{a}$ & $10,78 \mathrm{~b}$ & $5,69 \mathrm{a}$ & $2,12 \mathrm{a}$ & $3,26 \mathrm{a}$ & $13,57 \mathrm{a}$ & $3,50 \mathrm{a}$ & $5,50 \mathrm{a}$ & $9,77 \mathrm{a}$ & $6,28 \mathrm{a}$ \\
LN & $8,28 \mathrm{~b}$ & $6,61 \mathrm{~b}$ & $2,95 \mathrm{a}$ & $7,90 \mathrm{a}$ & $1,76 \mathrm{a}$ & $18,61 \mathrm{~b}$ & $16,30 \mathrm{a}$ & $7,14 \mathrm{a}$ & $9,61 \mathrm{a}$ & $11,95 \mathrm{a}$ & $16,47 \mathrm{a}$ \\
Neutro & $0,49 \mathrm{a}$ & $0,17 \mathrm{a}$ & $2,28 \mathrm{a}$ & $7,83 \mathrm{a}$ & $2,66 \mathrm{a}$ & $4,74 \mathrm{a}$ & $11,30 \mathrm{a}$ & $3,02 \mathrm{a}$ & $4,26 \mathrm{a}$ & $4,20 \mathrm{a}$ & $11,06 \mathrm{a}$ \\
\hline
\end{tabular}

Nota: Variâncias seguidas por letras iguais nas colunas não diferem entre si pelo teste F ao nível de 5\% de significância. Fonte: Elaborado pelos autores.

\section{Conclusões}

Baseado no teste $\mathrm{t}$ (Student) e no test $\mathrm{F}$, com nível de significância 5\%, é possível afirmar que o ENOS possui relação estatisticamente significativa com o número médio de ocorrência de ondas de frio em Santa Catarina somente nas estações de Itapiranga (na fase final do ENOS, pressupondo que em anos de El Niño tenhamos menores ocorrências de ondas de frio do que em anos com a atuação de La Niña); e na estação de Lages (durante as fases inicial e final, espera-se uma maior variação no número de ondas de frio durante a La Niña).

Já para os episódios de geadas, o ENOS possui relação comprovada estatisticamente, nas estações de Itajaí (maior variabilidade nas ocorrências de geada em ambas as fases do ENOS comparado aos anos neutros); São José e Chapecó (maior variabilidade nas ocorrências de geada em anos de La Niña) e Ituporanga (maior variabilidade nas ocorrências de geada em anos de El Niño).

\section{Agradecimentos}

Ao Programa de Pós-Graduação em Geografia - PPGG da UFSC, berço desta pesquisa por meio de uma dissertação já concluída e ao Laboratório de Climatologia da UFSC (LabClima).

\section{Bibliografia}

AGUIAR, D. MENDONÇA, M. (2004). Climatologia das geadas em Santa Catarina. In: SIMPÓSIO BRASILEIRO DE DESASTRES NATURAIS, 1., Florianópolis: GEDN/UFSC,2004. p. 762-773 (CD-ROM).

ALVES, M.P.A. Ondas de frio invernais em Santa Catarina e suas relações com as variabilidades climáticas de baixa frequência. Dissertação (Mestrado em Geografia) - Departamento de Geociências, Centro de Filosofia e Ciências Humanas, Universidade Federal de Santa Catarina. Florianópolis, 158 p.; 2016.

BARROS, V. R., A. M. Grimm, and M. E. Doyle.Relationship between temperature and circulation in southern South America and its influence from El Niño and La Niña events. J. Meteor. Soc. Japan, 80, 33-44,2002. 
BOYLE, J. S. (1986). Comparison of the synoptic conditions in midlatitudes accompanying cold surges over eastern Asia for the months of December 1974 and 1978. Part I: Monthly mean fields and individual events. Monthly Weather Review, n. 114 p. 903-930.

CASTRO, A.L.C.; CALHEIROS, L.B.; CUNHA, M.I.R.; BRINGEL, M.L.N.C. (1995). Manual de desastres naturais. Ministério do Planejamento e Orçamento, Secretaria Especial - Defesa Civil. Novembro, v. 1.

CPTEC/INPE - Centro de Previsão e Estudos Climáticos. Disponível em:< http://enos.cptec.inpe.br/> Acesso em: $23 \operatorname{dez} 2014$.

EPAGRI /CIRAM- EMPRESA DE PESQUISA AGROPECUÁRIA E EXTENSÃO RURAL DE SANTA CATARINA S.A / CENTRO DE INFORMAÇÕES DE RECURSOS AMBIENTAIS E DE HIDROMETEOROLOGIA DE SANTA CATARINA. Dados estações meteorológicas. Florianópolis, 2014.

ESCOBAR. G. (2007). Padrões Sinóticos Associados a Ondas de Frio na Cidade de São Paulo. Revista Brasileira de Meteorologia, v.22, n.2, 241-254.

FIRPO, M.A.F. (2008). Climatologia das ondas de frio e calor para o Rio Grande do Sul e sua Relação com o El Niño e La Niña. Dissertação apresentada de Pós-Graduação da Faculdade e Meteorologia da Universidade Federal de Pelotas. Pelotas, 120p.

FIRPO, M.A.F.; SANSIGOLO, C.A.E; ASSIS, S.V. Climatologia e variabilidade sazonal do número de ondas de calor e de frio no Rio Grande do Sul associadas ao ENOS. Revista Brasileira de Meteorologia, São Paulo, v.27, p.95-106, 2012.

GOLDEN GATE WEATHER SERVICES. ENSO Years based on Oceanic Niño Index (ONI). Disponível em:< http://ggweather.com/enso/oni.htm> Acesso em: 05 AGO. 2015.

GRIMM, A. M.; FERRAZ, S. E. T.; GOMES, J. Precipitation anomalies in Southern Brazil associated with El Niño and La Niña events. In: J. Climate, V.11, p. 2863 - 2880, 1998.

GRIMM, A. M. (2009). Variabilidade Interanual do Clima no Brasil. In: CAVALCANTI, I.F.A. et al (org). Tempo e Clima no Brasil. São Paulo: Oficina de texto.

GRIMM, A.M.; TOGATLIAN, I.M. Relação entre eventos EI Nino /La Nina e frequência de extremos frios e quentes de temperatura no Cone Sul da America do Sul. In: CONGRESSO BRASILEIRO DE METEOROLOGIA, 12., 2002, Foz do Iguaçu. Anais do... Foz do Iguaçu: SBMET, 2002. CD-ROM.

HAMMER, Ø, HARPER, DAT, RYAN, P.D. (2001). PASSADO: Estatísticas Paleontológicos pacote de software para educação e análise de dados. Palaeontologia Electronica 4 (1):. 9pp. Disponível em: $<$ http://palaeoelectronica.org/2001_1/past/issue1_01.htm> Acesso em: 05 jul. 2015.

KONRAD, C. E. (1996). Relationships between the intensity of cold-air outbreaks and the evolution of synoptic and planetary-scale features over North America. Monthly Weather Review, n. 124 p. 1067-1083.

MARENGO, J. A.; OLIVEIRA, G. S. Impactos do fenômeno La Niña no tempo e clima do Brasil: desenvolvimento e intensificação do La Niña 1998/1999. In: CONGRESSO BRASILEIRO DE METEOROLOGIA, 10, 1998, Brasília. Anais... Brasília, 1998. CD-ROM.

MENDONÇA, M.; ROMERO, H. (2012). ondas de frio, índices de oscilação e impactos socioambientais das variabilidades climáticas de baixa frequência na América do sul. Edição Especial - "Climatologia Geográfica", Revista Acta Geográfica (UFRR), v.2, p.185 - 185.

MINUZZI, B.M. (2010). Ocorrências de geada no estado de Santa Catarina. In: II SIMPÓSIO BRASILEIRO DE AGROPECUÁRIA SUSTENTAVÉL, 23 a 25 de Setembro de 2010 - Universidade Federal de Viçosa Viçosa/MG.

MONTEIRO, M. (2001). Caracterização climática do estado de Santa Catarina: uma abordagem dos principais sistemas atmosféricos que atuam durante o ano. Geosul. Revista do Departamento de Geociências da UFSC, Florianópolis, v. 16, n 31, p 69-78.

THOMÉ, V. M. R. et al. (1999). Zoneamento agroecológico e socioeconômico do Estado de Santa Catarina. Florianópolis: Epagri, CD-ROM. 


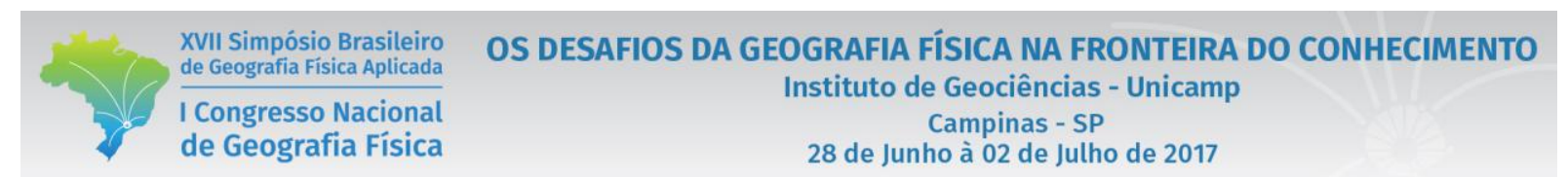

TRENBERTH, K. E. The definition of El Niño, Bulletin of the American Meteorological Society, v.78, n.12, p. 2771-2777, 1997.

WALSH, J. E.; PHILLIPS, A. S.; PORTIS, D. H.; CHAPMAN, W. L. Extreme cold outbreaks in the United States and Europe, 1948-99. Journal of Climate, n. 14, p. 2642-2658, 2001.

VAVRUS, S.; WALSH, J. E.; CHAPMAN, W. L.; PORTIS, D. (2006). The behavior of extreme cold air outbreaks under greenhouse warming. Int. J. Climatol., n. 26, p. 1133-1147. 